\title{
New Exact Jacobi Elliptic Function Solutions for the Coupled Schrödinger-Boussinesq Equations
}

\author{
Baojian Hong ${ }^{1,2}$ and Dianchen $\mathrm{Lu}^{1}$ \\ ${ }^{1}$ Faculty of Science, Jiangsu University, Zhenjiang, Jiangsu 212013, China \\ ${ }^{2}$ Department of Basic Courses, Nanjing Institute of Technology, Nanjing 211167, China \\ Correspondence should be addressed to Dianchen Lu; dclu@ujs.edu.cn
}

Received 18 July 2013; Accepted 2 September 2013

Academic Editor: Anjan Biswas

Copyright (C) 2013 B. Hong and D. Lu. This is an open access article distributed under the Creative Commons Attribution License, which permits unrestricted use, distribution, and reproduction in any medium, provided the original work is properly cited.

\begin{abstract}
A general algebraic method based on the generalized Jacobi elliptic functions expansion method, the improved general mapping deformation method, and the extended auxiliary function method with computerized symbolic computation is proposed to construct more new exact solutions for coupled Schrödinger-Boussinesq equations. As a result, several families of new generalized Jacobi elliptic function wave solutions are obtained by using this method, some of them are degenerated to solitary wave solutions and trigonometric function solutions in the limited cases, which shows that the general method is more powerful than plenty of traditional methods and will be used in further works to establish more entirely new solutions for other kinds of nonlinear partial differential equations arising in mathematical physics.
\end{abstract}

\section{Introduction}

Nonlinear partial differential equations (NLPDEs) are widely used to describe complex physical phenomena arising in the world around us and various fields of science. The investigation of exact solutions of NLPDEs plays an important role in the study of these phenomena such as the nonlinear dynamics and the mechanism behind the phenomena. With the development of soliton theory, many powerful methods for obtaining exact solutions of NLPDEs have been presented, such as homotopy perturbation method [1], nonperturbative method [2], homogeneous balance method [3], Bäcklund transformation [4], Darboux transformation [5], extended tanh-function method [6], extended $F$-expansion method [7], $G^{\prime} / G$ method [8], exp-function method [9], sine-cosine method [10], Jacobi elliptic function method [11], extended Riccati equation rational expansion method [12], extended auxiliary function method [13], and other methods [14, 15].

In $[16,17]$, Hong proposed a generalized Jacobi elliptic functions expansion method to obtain generalized exact solutions of NLPDEs. In [18], Hong and Lu proposed an improved general mapping deformation method which is more general than many other algebraic expansion methods
$[19,20]$. The solution procedure of this method, by the help of Matlab or Mathematica, is of utmost simplicity, and this method can be easily extended to all kinds of NLPDEs. In this work, we will propose the general algebraic method which contained the two methods [16-18] to obtain several new families of exact solutions for the coupled SchrödingerBoussinesq equations.

\section{Summary of the General Algebraic Method}

Consider a given nonlinear evolution equation with one physical field $u(x, t)$ in two variables $x$ and $t$

$$
P\left(u, u_{t}, u_{x}, u_{x x}, \ldots\right)=0 .
$$

We seek the following formal solutions of the given system by a new intermediate transformation:

$$
u(\xi)=\sum_{i=0}^{n} A_{i} \phi^{i}(\xi),
$$

where $A_{i}$ are constants to be determined later. $\xi=\xi(x, t)$ are arbitrary functions with the variables $x$ and $t$. The parameter 
$n$ can be determined by balancing the highest order derivative terms with the nonlinear terms in (1). And $\phi(\xi)$ is a solution of the following ordinary differential equation:

$$
\phi^{\prime 2}(\xi)=\sum_{i=0}^{4} a_{i} \phi^{i}(\xi) .
$$

Substituting (3) and (2) into (1) and setting the coefficients of $\phi^{i}(\xi)(i=0,1,2, \ldots)$ and $\phi^{j}(\xi) \sqrt{\sum_{i=0}^{4} a_{i} \phi^{i}(\xi)}(j=\ldots$, $-2,-1,0,1,2, \ldots)$ to zero yield a set of algebraic equations for $A_{i}, a_{i}$, and $\xi$. Using the Mathematica to solve the algebraic equations and substituting each of the solutions of the set, that is, each of the expressions of $\phi(\xi)$ into (2), we can get the solutions of (1). In order to obtain some new general solutions of (3), we assume that (3) has the following solutions:

$$
\phi(\xi)=c_{0}+c_{1} e(\xi)+c_{2} f(\xi)+c_{3} g(\xi)+c_{4} h(\xi),
$$

where $c_{i}=c_{i}(t)(i=0, \ldots, 4)$ are functions of $t$ to be determined later and the four functions $e=e(\xi), f=f(\xi)$, $g=g(\xi)$, and $h=h(\xi)$ are expressed as follows:

$$
\begin{array}{ll}
e=\frac{1}{p+q F+r F^{2}+l F^{\prime}}, & f=\frac{F}{p+q F+r F^{2}+l F^{\prime}}, \\
g=\frac{F^{2}}{p+q F+r F^{2}+l F^{\prime}}, & h=\frac{F^{\prime}}{p+q F+r F^{2}+l F^{\prime}}
\end{array}
$$

where $p, q, r$, and $l$ are arbitrary constants which ensure the denominator unequal to zero, and $F=F(\xi)$ is a solution of the following equations:

$$
\begin{aligned}
& F^{\prime 2}=A+B F^{2}+C F^{4}+2 D F+2 E F^{3} \\
& F^{\prime \prime}=B F+2 C F^{3}+D+3 E F^{2}
\end{aligned}
$$

where "' " denotes $d / d \xi$, “l” " denotes $d^{2} / d \xi^{2}, A, B, C, D$, and $E$ are arbitrary constants, and the four functions $e, f, g$, and $h$ satisfy the following relations:

$$
\begin{aligned}
& e^{\prime}=-q e h-2 r f h-l\left(D e^{2}+B e f+2 C f g+3 E f^{2}\right), \\
& f^{\prime}=p e h-r g h+l\left(A e^{2}+D e f-C g^{2}-E f g\right), \\
& g^{\prime}=q g h+2 p f h+l\left(2 A e f+3 D f^{2}+B f g+E g^{2}\right), \\
& h^{\prime}=(D p-A q) e^{2}+(B p-D q-2 A r) e f \\
& \quad+(2 C p+E q-B r) f g+3(E p-D r) f^{2}+(C q-E r) g^{2}, \\
& f^{2}=e g, \quad h^{2}=A e^{2}+B f^{2}+C g^{2}+2 D e f+2 E f g, \\
& p e+q f+r g+l h=1 .
\end{aligned}
$$

And $e, f, g$, and $h$ satisfy one of the following relations at the same time.
Family 1. When $p=0$

$$
\begin{aligned}
\left(C l^{2}-r^{2}\right) h^{2}= & -C+2 C l h-B r(1-l h-q f) e \\
& -A e^{2} r^{2}-2 D r^{2} e f+(2 C q-2 E r) f \\
& +(2 l E r-2 C l q) f h+\left(2 E q r-C q^{2}\right) f^{2}
\end{aligned}
$$

Family 2. When $q=0$

$$
\begin{aligned}
\left(C l^{2}-r^{2}\right) h^{2}= & 2 C(l h+p e-p l e h)+2 E r(l h-1) f \\
& -B r(1-l h-p e) e-\left(C p^{2}+A r^{2}\right) e^{2} \\
& +\left(2 E p r-2 D r^{2}\right) e f-C .
\end{aligned}
$$

Family 3. When $r=0$

$$
\begin{aligned}
C l^{2} g^{2}= & 1-2 E l^{2} f g-2 p e+\left(p^{2}-A l^{2}\right) e^{2} \\
& -2 q f+2\left(p q-D l^{2}\right) e f+\left(q^{2}-B l^{2}\right) e g .
\end{aligned}
$$

Family 4. When $l=0$

$$
\begin{aligned}
r^{2} h^{2}= & C-2 C p e+(2 E r-2 C q) f+\left(C p^{2}+A r^{2}\right) e^{2} \\
& +\left(C q^{2}-2 E q r+B r^{2}\right) e g \\
& +\left(2 C p q-2 E p r+2 D r^{2}\right) e f .
\end{aligned}
$$

Substituting (4), (7) along with (8a)-(8d) into (3) separately yields four families of polynomial equations for $e, f, g$, and $h$. Setting the coefficients of $e^{i}, e^{i} f, e^{i} g, e^{i} h, e^{i} f g, e^{i} f h$, and $e^{i} g h(i=0,1,2, \ldots)$ to zero yields a set of overdetermined differential equations (ODEs) in $p, q, r, l, a_{i}$, and $c_{i}(i=$ $0,1,2,3,4), A, B, C, D, E$, and $\xi(x, t)$, solving the ODEs by Mathematica and $\mathrm{Wu}$ elimination, we can obtain many exact solutions of (3) according to (4), (5), (6). If we let $c_{0}=c_{1}=$ $c_{3}=c_{4}=0, c_{2}=1, p=1, q=r=l=0, a_{0}=A, a_{1}=2 D$, $a_{2}=B, a_{3}=2 E$, and $a_{4}=C$, we have $\varphi(\xi)=F(\xi)$; our method contains the improved general mapping deformation method [18].

Remark 1. Our method proposed here is more general than the $G^{\prime} / G$ method [8], the extended Riccati equation rational expansion method [12], the extended auxiliary function method [13], the generalized Jacobi elliptic functions expansion method $[16,17]$, and many other algebra expansion methods $[6,7,11,18-21]$. 
Remark 2. Equations (2) and (3) can be extended to the following forms:

$$
\begin{aligned}
u(\xi)= & \sum_{i=0}^{n} A_{i}(t) \phi^{i}(\xi)+\sum_{i=-n}^{-1} A_{n-i}(t) \phi^{i}(\xi) \\
& +\sum_{i=-n}^{n} B_{i}(t) \phi^{i}(\xi) \phi^{\prime}(\xi) \\
\phi^{\prime 2}(\xi)= & \sum_{i=0}^{N} a_{i}(t) \phi^{i}(\xi)
\end{aligned}
$$

where $N$ can be an arbitrary positive integer. $n$ is usually a positive integer. If $n$ is a fraction or a negative integer, we make the following transformation:

(a) when $n=d / c$ is a fraction, we let $u(\xi)=v^{d / c}(\xi)$, then return to determine the balance constant $n$ again;

(b) when $n$ is a negative integer, we suppose $u(\xi)=v^{n}(\xi)$, then return to determine the balance constant $n$ again.

Remark 3. Notice that

$$
\begin{aligned}
\left.\left.F_{1}(\xi)\right|_{(A, B, C, D, E)_{1}} \longrightarrow \varphi_{1}(\xi)\right|_{\left(a_{0}, a_{1}, a_{2}, a_{3}, a_{4}\right)_{1}} \\
=\left.\left.F_{2}(\xi)\right|_{(A, B, C, D, E)_{2}} \longrightarrow \varphi_{2}(\xi)\right|_{\left(a_{0}, a_{1}, a_{2}, a_{3}, a_{4}\right)_{2}} \\
\quad=\left.F_{3}(\xi)\right|_{(A, B, C, D, E)_{3}} \longrightarrow \cdots .
\end{aligned}
$$

We find a meanful conclusion that this general method implies a BT of (1) with the compatible conditions (4), (5), (6), (7), and (8a)-(8d).

In the following, we will use this method to solve the Schrödinger-Boussinesq equations.

\section{Exact Solutions to the Coupled Schrödinger- Boussinesq Equations}

We consider the coupled Schrödinger-Boussinesq equations [21-28]

$$
\begin{gathered}
i E_{t}+E_{x x}+\alpha E-N E=0 \\
3 N_{t t}-N_{x x x x}+3\left(N^{2}\right)_{x x}+\beta N_{x x}-\left(|E|^{2}\right)_{x x}=0 .
\end{gathered}
$$

These equations are known to describe various physical processes in laser and plasma, such as formation, Langmuir field amplitude, intense electromagnetic waves, and modulational instabilities [22-25]. The problem of the complete integrability of this system has been studied by Chowdhury et al. from the point of view of Painlevé analysis [25]. The solitary wave solutions for system (11) have been obtained in $[26,27]$. The Jacobi doubly periodic wave solutions and a range of other solutions for this system have been investigated in $[21,28]$. We are interested in searching new generalized Jacobi elliptic function solutions for (11) by using our method.
We consider the following transformations:

$$
\begin{gathered}
E(x, t)=E(\xi)=u(\xi) e^{i\left(k x+\omega t+\xi_{00}\right)}, \\
N(x, t)=N(\xi)=v(\xi), \\
\xi=a x-2 a k t+\xi_{0},
\end{gathered}
$$

where $a$ and $k$ are constants to be determined later and $\xi_{0}$ and $\xi_{00}$ are arbitrary constants.

Substituting (12) and (13) into (11) and integrating the second equation of system (11) twice, we have

$$
\begin{gathered}
a^{2} u^{\prime \prime}+\left(\alpha-\omega-k^{2}\right) u-u v=0, \\
a^{2} v^{\prime \prime}+u^{2}-\left(12 k^{2}+\beta\right) v-3 v^{2}=0 .
\end{gathered}
$$

By balancing the highest derivative term with the nonlinear terms in (14), we obtain $n=2$. Therefore, we assume that (14) have the following solutions:

$$
\begin{gathered}
u(\xi)=A_{0}+A_{1} \varphi(\xi)+A_{2} \varphi^{2}(\xi), \\
v(\xi)=B_{0}+B_{1} \varphi(\xi)+B_{2} \varphi^{2}(\xi) .
\end{gathered}
$$

Substituting (3), (13), and (15) into (14) and setting the coefficients of $\phi^{i}(\xi)(i=0,1,2, \ldots)$ and $\varphi^{j}(\xi) \sqrt{\sum_{i=0}^{4} a_{i} \varphi^{i}(\xi)}(j=$ $\ldots,-2,-1,0,1,2, \ldots)$ to zero yield a set of over-determined equations (ODEs) for $A_{i}, k, \omega$, and $a_{i}$. After solving the ODEs by Mathematica, we could determine the following solutions.

Family 1.

$$
\begin{aligned}
& A_{0}= \pm a^{2} \sqrt{\frac{-a_{1} a_{3}-8 a_{0} a_{4}}{2}}, \\
& A_{1}= \pm \frac{2 a^{2} a_{4}}{a_{3}} \sqrt{-2 a_{1} a_{3}-16 a_{0} a_{4}}, \quad A_{2}=0, \\
& B_{0}=0, \quad B_{1}=a^{2} a_{3}, \quad B_{2}=2 a^{2} a_{4}, \\
& \omega=-\frac{a^{2}\left(a_{3}^{4}-80 a_{1} a_{3} a_{4}^{2}-128 a_{0} a_{4}^{3}\right)}{48 a_{3}^{2} a_{4}}+\alpha+\frac{\beta}{12}, \\
& k=\frac{\varepsilon}{4} \sqrt{\frac{a^{2}\left(a_{3}^{4}+16 a_{1} a_{3} a_{4}^{2}-128 a_{0} a_{4}^{3}\right)-4 a_{3}^{2} a_{4} \beta}{3 a_{3}^{2} a_{4}},} \\
& \varepsilon^{2}=1, \quad a_{2}=\frac{a_{3}^{2}}{4 a_{4}}+\frac{2 a_{1} a_{4}}{a_{3}} .
\end{aligned}
$$

Family 2.

$$
\begin{aligned}
& A_{0}= \pm \sqrt{2} B_{0}, \quad A_{1}=0, \quad A_{2}= \pm 6 \sqrt{2} a^{2} a_{4}, \\
& B_{0}=\text { const }, \quad B_{1}=0, \quad B_{2}=6 a^{2} a_{4}, \\
& a_{1}=a_{3}=0, \quad \omega=\frac{1}{12}\left(44 a^{2} a_{2}-22 B_{0}+12 \alpha+\beta\right), \\
& k=\varepsilon \sqrt{\frac{4 a^{2} a_{2}-2 B_{0}-\beta}{12}}, \quad \varepsilon^{2}=1 .
\end{aligned}
$$




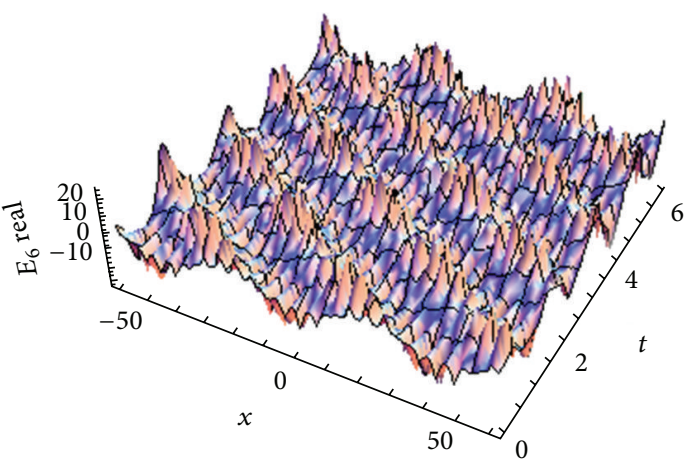

(a)

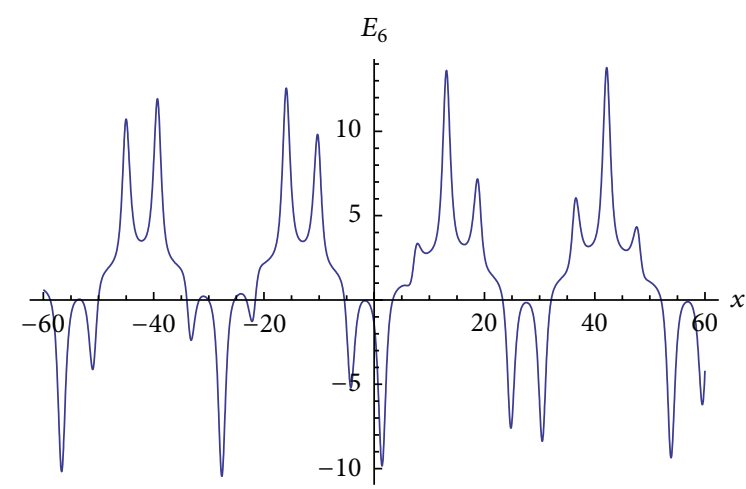

(b)

FIgURE 1: The real part of $E_{6}$ when $a=\alpha=1, \beta=-1, \xi_{0}=0$, and $m=0.9$ and a plane graph when $t=0$.

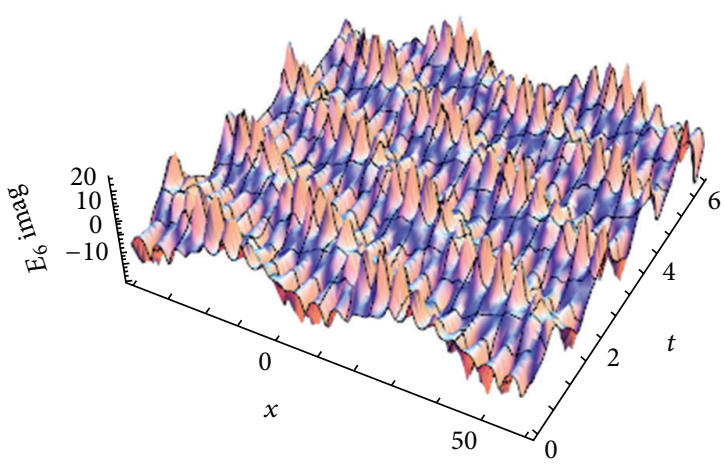

(a)

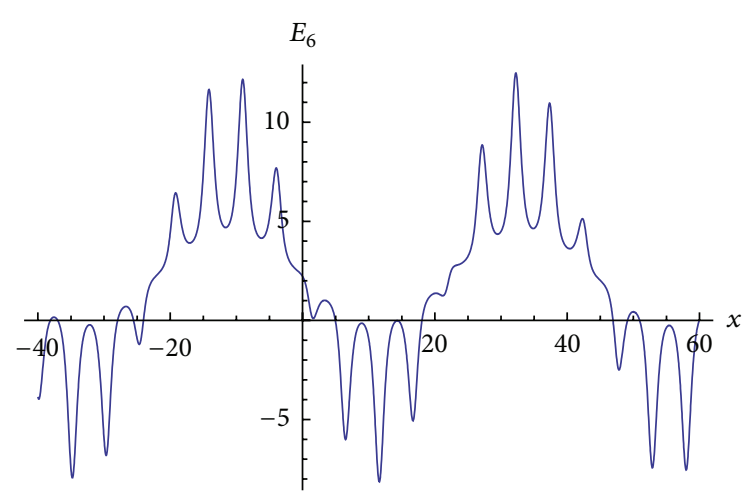

(b)

Figure 2: The imaginary part of $E_{6}$ when $a=\alpha=1, \beta=-1, \xi_{0}=0$, and $m=0.9$ and a plane graph when $t=0$.

Substituting (4), (5), (6), (7) along with (8a)-(8d) and (16) into (14) separately yields an ODEs; after solving the ODEs by Mathematica and Wu elimination, we can obtain the following solutions of (14) according to (4), (5), (6), (16).

Case 1.

$$
\begin{gathered}
A=a_{0_{1}}=\frac{1}{4}\left(1-m^{2}\right), \quad B=a_{2_{1}}=2-m^{2}, \\
C=a_{4_{1}}=1, \quad D=\frac{1}{2} a_{1_{1}}=\frac{1}{2}\left(m^{2}-1\right), \\
E=\frac{1}{2} a_{3_{1}}=-1, \quad 0 \leq m \leq 1, \\
p=1, \quad q=r=l=0, \\
c_{0}=c_{1}=c_{3}=c_{4}=0, \quad c_{2}=1, \\
\varphi_{1}\left(\xi_{1}\right)=F_{1}\left(\xi_{1}\right)=\frac{\mathrm{cn} \xi_{1}}{ \pm 1 \pm \operatorname{sn} \xi_{1}+\mathrm{cn} \xi_{1}}, \\
\xi_{1}=a x-2 a k_{1} t+\xi_{0} .
\end{gathered}
$$

Case 2.

$$
\begin{gathered}
A=a_{0_{2}}=\frac{1}{4}, \quad B=a_{2_{2}}=2-m^{2}, \\
C=a_{4_{2}}=1-m^{2}, \quad D=\frac{1}{2} a_{1_{2}}=\frac{1}{2},
\end{gathered}
$$

$$
\begin{aligned}
\varphi_{2}\left(\xi_{2}\right)=F_{2}\left(\xi_{2}\right) & =\frac{\operatorname{sn} \xi_{2}}{ \pm 1-\operatorname{sn} \xi_{2} \pm \operatorname{cn} \xi_{2}}, \\
\xi_{2} & =a x-2 a k_{2} t+\xi_{0} .
\end{aligned}
$$

Case 3.

$$
\begin{gathered}
A=a_{0_{3}}=-\left(c_{0}^{2}-1\right)\left(1+\left(c_{0}^{2}-1\right) m^{2}\right), \\
B=a_{2_{3}}=\left(2-6 c_{0}^{2}\right) m^{2}-1, \quad C=a_{4_{3}}=-m^{2}, \\
D=\frac{1}{2} a_{1_{3}}=c_{0}-2 c_{0} m^{2}+2 c_{0}^{3} m^{2}, \\
E=\frac{1}{2} a_{3_{3}}=2 c_{0} m^{2}, \quad 0 \leq m \leq 1, \\
p=1, \quad q=r=l=0, \\
c_{0}=\text { const, } \quad c_{1}=c_{3}=c_{4}=0, \quad c_{2}=1, \\
\varphi_{3}\left(\xi_{3}\right)=F_{3}\left(\xi_{3}\right)=c_{0}+\mathrm{cn}^{2} \xi_{3}, \quad \xi_{3}=a x-2 a k_{3} t+\xi_{0} .
\end{gathered}
$$




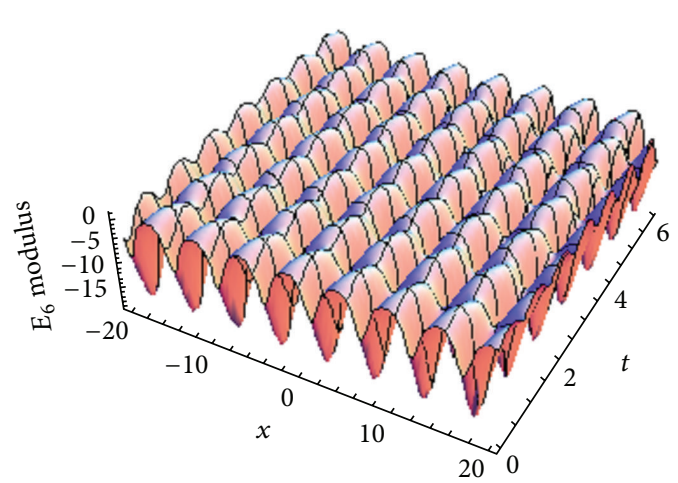

(a)

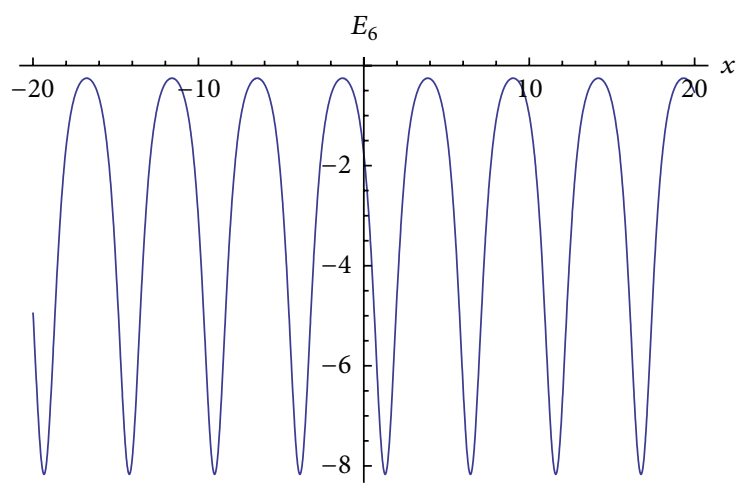

(b)

FIgURE 3: The modulus of $E_{6}$ when $a=\alpha=1, \beta=-1, \xi_{0}=0$, and $m=0.9$ and a plane graph when $t=0$.

Case 4.

$$
\begin{gathered}
A=1, \quad B=-m^{2}-1, \quad C=m^{2}, \\
D=E=0, \quad F=\operatorname{sn}, \quad 0 \leq m \leq 1, \\
a_{0_{4}}=1, \quad a_{1_{4}}=\mp 4 \sqrt{2(1+m) \sqrt{m}}, \\
a_{3_{4}}= \pm 2 \sqrt{2(1+m) \sqrt{m}}\left(1+6 m+m^{2}-4(1+m) \sqrt{m}\right), \\
a_{2_{4}}=12(1+m) \sqrt{m}-6 m-m^{2}-1, \\
a_{4_{4}}=8 m(m+1)^{2}-2(1+m)\left(1+m^{2}+6 m\right) \sqrt{m}, \\
p=1, \quad q= \pm \sqrt{2(1+m) \sqrt{m}}, \quad r=m, \\
l=0, \quad c_{0}=c_{1}=c_{3}=c_{4}=0, \\
\varphi_{4}\left(\xi_{4}\right)=\frac{c^{2} \xi_{4}}{1 \pm \sqrt{2(1+m) \sqrt{m}},} \\
\xi_{4}=a x-2 a k_{4}+m s^{2} \xi_{4}
\end{gathered}
$$

Case 5.

$$
\begin{gathered}
A=1-m^{2}, \quad B=2 m^{2}-1, \\
C=-m^{2}, \quad D=E=0, \quad 0 \leq m \leq 1, \\
F=\mathrm{cn} \xi \quad a_{0_{5}}=1, \quad a_{1_{5}}=-4, \\
a_{2_{5}}=8-4 m^{2}, \quad a_{3_{5}}=8 m^{2}-8, \quad a_{4_{5}}=4-4 m^{2}, \\
p=0, \quad q=1, \quad r=0, \quad l= \pm 1, \\
c_{0}=c_{1}=c_{3}=c_{4}=0, \quad c_{2}=1, \\
\varphi_{5}\left(\xi_{5}\right)=\frac{\mathrm{cn} \xi_{5}}{\mathrm{cn} \xi_{5} \mp \operatorname{sn} \xi_{5} \operatorname{dn} \xi_{5}}, \quad \xi_{5}=a x-2 a k_{5} t+\xi_{0} .
\end{gathered}
$$

Case 6.

$$
\begin{gathered}
A=m^{2}-1, \quad B=2-m^{2}, \quad C=-1, \\
D=E=0, \quad 0 \leq m \leq 1, \\
F=\operatorname{dn} \xi \quad a_{0_{6}}=1, \quad a_{1_{6}}=-4 m, \\
a_{2_{6}}=8 m^{2}-4, \quad a_{3_{6}}=8 m-8 m^{3}, \quad a_{4_{6}}=4 m^{4}-4 m^{2}, \\
p=0, \quad q=m, \quad r=0, \quad l= \pm 1, \\
c_{0}=c_{1}=c_{3}=c_{4}=0, \quad c_{2}=1, \\
\varphi_{6}\left(\xi_{6}\right)=\frac{\operatorname{dn} \xi_{6}}{\operatorname{dn} \xi_{6} \mp m^{2} \operatorname{sn} \xi_{6} \mathrm{cn} \xi_{6}}, \quad \xi_{6}=a x-2 a k_{6} t+\xi_{0} .
\end{gathered}
$$

Case 7.

$$
\begin{gathered}
A=\frac{C_{1} C_{3} q-5 C_{3}^{2} q^{3}+\varepsilon\left(C_{1}+3 C_{3} q^{2}\right) \sqrt{C_{3}\left(3 C_{3} q^{2}-2 C_{1}\right)}}{4 C_{3}}, \\
B=0, \quad C=0, \quad 2 D=C_{1}, \quad 2 E=C_{3}, \\
F=\wp\left(\frac{\sqrt{C_{3}}}{2} \xi, \frac{-4 C_{1}}{C_{3}}, \frac{-4 M}{C_{3}}\right), \\
a_{0_{7}}=0, \quad a_{1_{7}}=C_{3}, \quad a_{2_{7}}=-3 C_{3} q, \\
a_{4_{7}}=\left(\begin{array}{l}
-3 C_{1} C_{3} q-9 C_{3}^{2} q^{3} \\
\quad
\end{array}\right. \\
\left.\quad \times\left(C_{1}+3 C_{3} q^{2}\right) \sqrt{C_{3}\left(3 C_{3} q^{2}-2 C_{1}\right)}\right) \\
\times\left(4 C_{3}\right)^{-1},
\end{gathered}
$$




$$
\begin{gathered}
\varepsilon=\operatorname{sgn}\left[C_{1}+3 C_{3} q^{2}\right], \quad p=0, \quad q=\text { const, } \\
r=1, \quad l=0, \quad c_{0}=c_{1}=c_{3}=c_{4}=0, \quad c_{2}=1, \\
\varphi_{7}\left(\xi_{7}\right)=\frac{1}{q+\wp\left(\left(\sqrt{C_{3}} / 2\right) \xi_{7},-4 C_{1} / C_{3},-4 M / C_{3}\right)}, \\
\xi_{7}=a x-2 a k_{7} t+\xi_{0} .
\end{gathered}
$$

We obtain the following solutions of (11) according to (4), (5), (12), (13), (15), and (16) and Cases 1-7:

$$
\begin{aligned}
& E_{i}(x, t)= {\left[ \pm a^{2} \sqrt{\frac{-a_{1_{i}} a_{3_{i}}-8 a_{0_{i}} a_{4_{i}}}{2}}\right.} \\
&\left. \pm \frac{2 a^{2} a_{4_{i}}}{a_{3_{i}}} \sqrt{-2 a_{1_{i}} a_{3_{i}}-16 a_{0_{i}} a_{4_{i}}} \varphi_{i}\left(\xi_{i}\right)\right] \\
& \times e^{i\left(k_{i} x+\omega_{i} t+\xi_{00}\right)}, \\
& N_{i}(x, t)= a^{2} a_{3_{i}} \varphi_{i}\left(\xi_{i}\right)+2 a^{2} a_{4_{i}} \varphi_{i}^{2}\left(\xi_{i}\right), \\
& \xi_{i}=a x-2 a k_{i} t+\xi_{0}, \quad(i=1, \ldots, 7),
\end{aligned}
$$

where $a$ is an arbitrary constant, $a_{0_{i}}, a_{1_{i}}, a_{2_{i}}, a_{3_{i}}$, and $a_{4_{i}}$ are defined as Cases 1-7, and $\omega_{i}$ and $k_{i}$ are defined as follows:

$$
\begin{array}{r}
\omega_{i}=-\frac{a^{2}\left(a_{3_{i}}^{4}-80 a_{1_{i}} a_{3_{i}} a_{4_{i}}^{2}-128 a_{0_{i}} a_{4_{i}}^{3}\right)}{48 a_{3_{i}}^{2} a_{4_{i}}}+\alpha+\frac{\beta}{12}, \\
k_{i}=\frac{\varepsilon}{4} \sqrt{\frac{a^{2}\left(a_{3_{i}}^{4}+16 a_{1_{i}} a_{3_{i}} a_{4_{i}}^{2}-128 a_{0_{i}} a_{4_{i}}^{3}\right)-4 a_{3_{i}}^{2} a_{4_{i}} \beta}{3 a_{3_{i}}^{2} a_{4_{i}}}}, \\
\varepsilon^{2}=1, \quad(i=1, \ldots, 7) .
\end{array}
$$

With the similar process,substituting (4), (5), (6), and (7) along with (8a)-(8d) and (17) into (14) separately yields ODEs; after solving the ODEs by Mathematica and $\mathrm{Wu}$ elimination, we can obtain the following solutions of (11) according to (4), (5), (12), (13), (15), and (17) and the solutions of (3) mentioned in [29]:

$$
\begin{aligned}
& E_{j}(x, t)=\left[ \pm \sqrt{2} B_{0} \pm 6 \sqrt{2} a^{2} a_{4_{j}} \varphi_{j}^{2}\left(\xi_{j}\right)\right] e^{i\left(k_{j} x+\omega_{j} t+\xi_{00}\right)}, \\
& \quad(j=0,1,2, \ldots), \\
& N_{j}(x, t)=B_{0}+6 a^{2} a_{4_{j}} \varphi_{j}^{2}\left(\xi_{j}\right), \quad \xi_{j}=a x-2 a k_{j} t+\xi_{0}, \\
& \omega_{j}=\frac{1}{12}\left(44 a^{2} a_{2_{j}}-22 B_{0}+12 \alpha+\beta\right), \\
& k_{j}=\varepsilon \sqrt{\frac{4 a^{2} a_{2_{j}}-2 B_{0}-\beta}{12}}, \quad \varepsilon^{2}=1 .
\end{aligned}
$$

Remark 4. If we let $a_{0_{1.1}}=1-m^{2}, a_{2_{1.1}}=2 m^{2}-1, a_{4_{1.1}}=-m^{2}$, $a_{1_{1.1}}=a_{3_{1.1}}=0$, and $\varphi_{1.1}\left(\xi_{1.1}\right)=\mathrm{cn} \xi_{1.1}$, then we have

$E_{1.1}(x, t)=\left[ \pm \sqrt{2} B_{0} \mp 6 \sqrt{2} a^{2} m^{2} \mathrm{cn}^{2}\left(\xi_{1.1}\right)\right] e^{i\left(k_{1} x+\omega_{1} t+\xi_{00}\right)}$,

$N_{1.1}(x, t)=B_{0}-6 a^{2} m^{2} \mathrm{cn}^{2}\left(\xi_{1.1}\right), \quad \xi_{1.1}=a x-2 a k_{1.1} t+\xi_{0}$,

$\omega_{1.1}=\frac{1}{12}\left(44 a^{2}\left(2 m^{2}-1\right)-22 B_{0}+12 \alpha+\beta\right)$,

$k_{1.1}=\varepsilon \sqrt{\frac{4 a^{2}\left(2 m^{2}-1\right)-2 B_{0}-\beta}{12}}, \quad \varepsilon^{2}=1$.

If we let $a=(1 / 2) \sqrt{b_{0}}, m=1$, or $a=(1 / 2) \sqrt{\left(1 / 2 m^{2}-1\right) b_{0}}$, then solution $\left(E_{1.1}(x, t), N_{1.1}(x, t)\right)$ is in full agreement with the solution $E_{1}, N_{1}$ and $E_{2}, N_{2}$ mentioned in [21].

Remark 5. The seven types of explicit solutions we obtained here to (11) are not shown in the previous literature to our knowledge. They are new exact solutions of (11). Notice that $\operatorname{sn} \xi \rightarrow \tanh \xi, \operatorname{cn} \xi \rightarrow \operatorname{sech} \xi$, and $\operatorname{dn} \xi \rightarrow \operatorname{sech} \xi$ when the modulus $m \rightarrow 1$, and $\operatorname{sn} \xi \rightarrow \sin \xi, \operatorname{cn} \xi \rightarrow \cos \xi$, and $\operatorname{dn} \xi \rightarrow 1$ when the modulus $m \rightarrow 0$. Solutions $E_{i}, N_{i}(i=$ $1, \ldots, 6)$ are degenerated to solitary wave solutions when the modulus $m \rightarrow 1$ and to triangular functions solutions when the modulus $m \rightarrow 0$.

We can give the numerical simulation of $E_{6}$ to show their physical properties (see Figures 1, 2, and 3).

\section{Conclusion}

In this paper, we succeed to propose a general algebraic method approach for finding new exact solutions of the nonlinear evolution equations. By using this method and computerized symbolic computation, we have found abundant new exact solutions for the coupled SchrödingerBoussinesq equations (11). More importantly, our method is much simpler and powerful to find new solutions to various kinds of nonlinear evolution equations, such as $\mathrm{KdV}$ equation, Boussinesq equation, and zakharov equation. We believe that this method should play an important role for finding exact solutions in the mathematical physics.

\section{Acknowledgments}

This work is supported by the National Natural Science Foundation of China (Grant no. 61070231), the Outstanding Personal Program in Six Fields of Jiangsu (Grant no. 2009188), the Graduate Student Innovation Project of Jiangsu Province (Grant no. CXLX13_673), and the General Program of Innovation Foundation of Nanjing Institute of Technology (Grant no. CKJB201218).

\section{References}

[1] J. He, "Homotopy perturbation method for bifurcation of nonlinear problems," International Journal of Nonlinear Sciences and Numerical Simulation, vol. 6, no. 2, pp. 207-208, 2005. 
[2] A. Lacour, J. A. Oller, and U.-G. Meißner, "Non-perturbative methods for a chiral effective field theory of finite density nuclear systems," Annals of Physics, vol. 326, no. 2, pp. 241-306, 2011.

[3] A. S. A. Rady, E. S. Osman, and M. Khalfallah, "The homogeneous balance method and its application to the BenjaminBona-Mahoney (BBM) equation," Applied Mathematics and Computation, vol. 217, no. 4, pp. 1385-1390, 2010.

[4] D. Lu and B. Hong, "Bäcklund transformation and $n$-solitonlike solutions to the combined KdV-Burgers equation with variable coefficients," International Journal of Nonlinear Science, vol. 2, no. 1, pp. 3-10, 2006.

[5] H.-C. Hu, X.-Y. Tang, S.-Y. Lou, and Q.-P. Liu, "Variable separation solutions obtained from Darboux transformations for the asymmetric Nizhnik-Novikov-Veselov system," Chaos, Solitons and Fractals, vol. 22, no. 2, pp. 327-334, 2004.

[6] H. A. Abdusalam, "On an improved complex tanh-function method," International Journal of Nonlinear Sciences and Numerical Simulation, vol. 6, no. 2, pp. 99-106, 2005.

[7] M. A. Abdou, "An improved generalized F-expansion method and its applications," Journal of Computational and Applied Mathematics, vol. 214, no. 1, pp. 202-208, 2008.

[8] S. Kumar, K. Singh, and R. K. Gupta, "Coupled Higgs field equation and Hamiltonian amplitude equation: lie classical approach and (G'/G)-expansion method," Parmana, vol. 79, pp. 41-60, 2012.

[9] A. Ebaid, "An improvement on the Exp-function method when balancing the highest order linear and nonlinear terms," Journal of Mathematical Analysis and Applications, vol. 392, no. 1, pp. 15, 2012.

[10] E. M. E. Zayed and M. A. M. Abdelaziz, "Exact solutions for the nonlinear Schrödinger equation with variable coefficients using the generalized extended tanh-function, the sine-cosine and the exp-function methods," Applied Mathematics and Computation, vol. 218, no. 5, pp. 2259-2268, 2011.

[11] W. Huang and Y. Liu, "Jacobi elliptic function solutions of the Ablowitz-Ladik discrete nonlinear Schrödinger system," Chaos, Solitons and Fractals, vol. 40, no. 2, pp. 786-792, 2009.

[12] W. Li and H. Zhang, "A new generalized compound Riccati equations rational expansion method to construct many new exact complexiton solutions of nonlinear evolution equations with symbolic computation," Chaos, Solitons and Fractals, vol. 39, no. 5, pp. 2369-2377, 2009.

[13] Y. Feng and H.-Q. Zhang, "A new auxiliary function method for solving the generalized coupled Hirota-Satsuma KdV system," Applied Mathematics and Computation, vol. 200, no. 1, pp. 283288, 2008.

[14] A. Biswas, E. V. Krishnan, P. Suarez, A. H. Kara, and S. Kumar, "Solitary waves and conservation laws of Bona-Chen equation," Indian Journal of Physics, vol. 87, pp. 169-175, 2013.

[15] E. V. Krishnan, G. Ebadi, and A. Biswas, "Solutions of a coupled wave equation," University Polytechnica of Bucharest Scientific Bulletin A, vol. 75, pp. 57-68, 2013.

[16] B. Hong, "New Jacobi elliptic functions solutions for the variable-coefficient MKdV equation," Applied Mathematics and Computation, vol. 215, no. 8, pp. 2908-2913, 2009.

[17] B. Hong, "New exact Jacobi elliptic functions solutions for the generalized coupled Hirota-Satsuma KdV system," Applied Mathematics and Computation, vol. 217, no. 2, pp. 472-479, 2010.
[18] B. Hong and D. Lu, "New Jacobi elliptic function-like solutions for the general KdV equation with variable coefficients," Mathematical and Computer Modelling, vol. 55, no. 3-4, pp. 1594-1600, 2012.

[19] Z. T. Fu, S. D. Liu, S. K. Liu, and Q. Zhao, "New exact solutions to KdV equations with variable coefficients or forcing," Applied Mathematics and Mechanics, vol. 25, no. 1, pp. 73-79, 2004.

[20] B. Hong and D. Lu, "New exact solutions for the generalized variable-coefficient Gardner equation with forcing term," Applied Mathematics and Computation, vol. 219, no. 5, pp. 27322738, 2012.

[21] Y. C. Hon and E. G. Fan, "A series of exact solutions for coupled Higgs field equation and coupled Schrödinger-Boussinesq equation," Nonlinear Analysis: Theory, Methods \& Applications A, vol. 71, no. 7-8, pp. 3501-3508, 2009.

[22] N. N. Rao and P. K. Shukla, "Coupled Langmuir and ionacoustic waves in two-electron temperature plasmas," Physics of Plasmas, vol. 4, no. 3, pp. 636-645, 1997.

[23] N. L. Shatashvili and N. N. Rao, "Localized nonlinear structures of intense electromagnetic waves in two-electron-temperature electron-positron-ion plasmas," Physics of Plasmas, vol. 6, no. 1, pp. 66-71, 1999.

[24] P. Saha, S. Banerjee, and A. R. Chowdhury, "Normal form analysis and chaotic scenario in a Schrödinger-Boussinesq system," Chaos, Solitons and Fractals, vol. 14, no. 1, pp. 145-153, 2002.

[25] A. R. Chowdhury, B. Dasgupta, and N. N. Rao, "Painléve analysis and Backlund transformations for coupled generalized Schrödinger-Boussinesq system," Chaos, Solitons and Fractals, vol. 9, no. 10, pp. 1747-1753, 1998.

[26] Y. Hase and J. Satsuma, "An N-soliton solution for the nonlinear Schrödinger equation coupled to the Boussinesq equation," Journal of the Physical Society of Japan, vol. 57, no. 3, pp. 679$682,1988$.

[27] X. Hu, B. Guo, and H. Tam, "Homoclinic orbits for the coupled Schrödinger-Boussinesq equation and coupled higgs equation," Journal of the Physical Society of Japan, vol. 72, no. 1, pp. 189-190, 2003.

[28] W. Li, E. Yomba, and H. Zhang, "New exact solutions for some coupled nonlinear partial differential equations using extended coupled sub-equations expansion method," Applied Mathematics and Computation, vol. 217, no. 21, pp. 8468-8481, 2011.

[29] A. Ebaid and E. H. Aly, "Exact solutions for the transformed reduced Ostrovsky equation via the F-expansion method in terms of Weierstrass-elliptic and Jacobian-elliptic functions," Wave Motion, vol. 49, no. 2, pp. 296-308, 2012. 


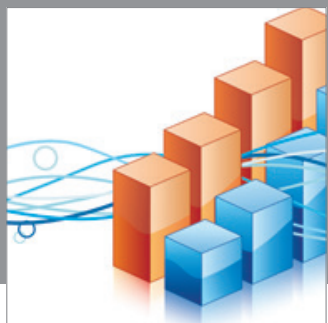

Advances in

Operations Research

mansans

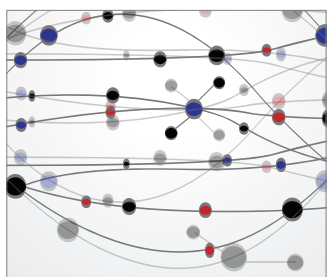

The Scientific World Journal
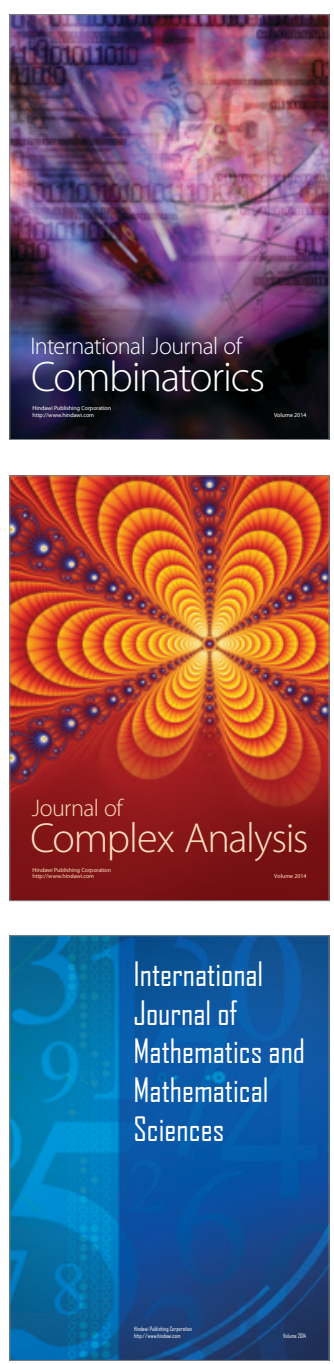
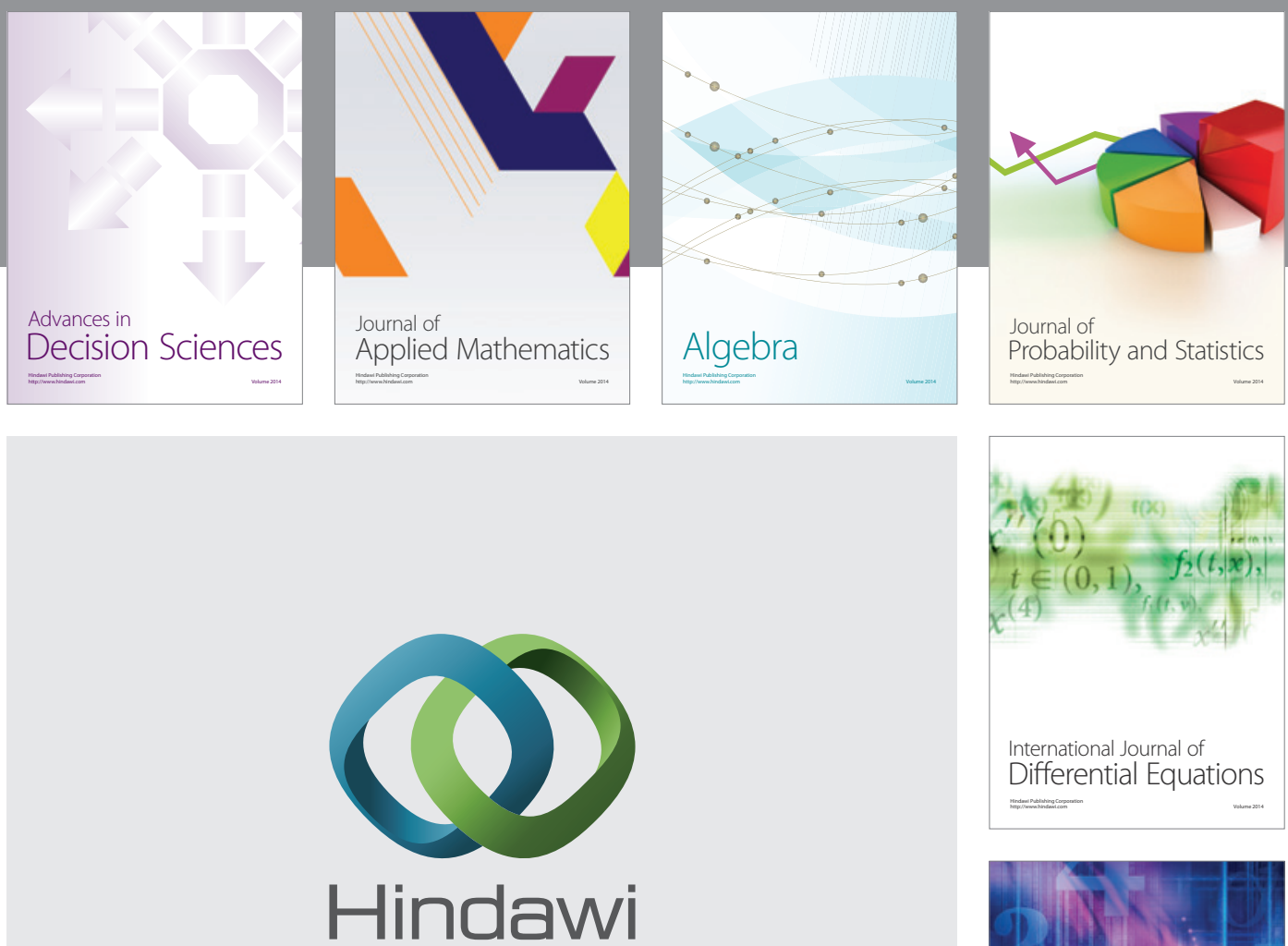

Submit your manuscripts at http://www.hindawi.com
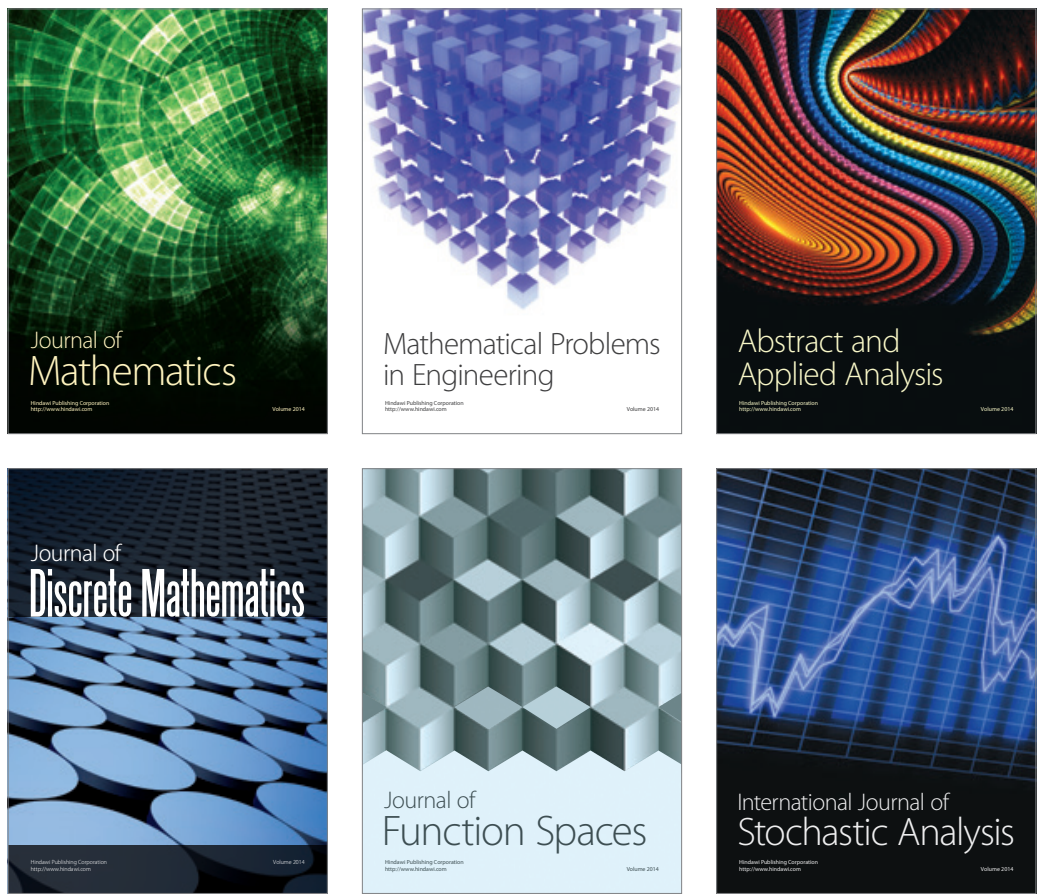

Journal of

Function Spaces

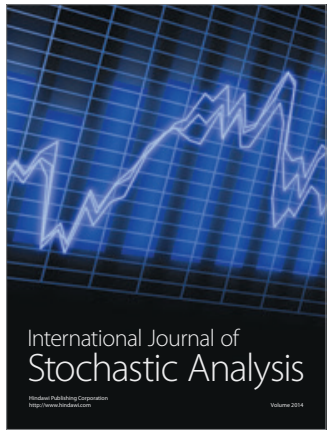

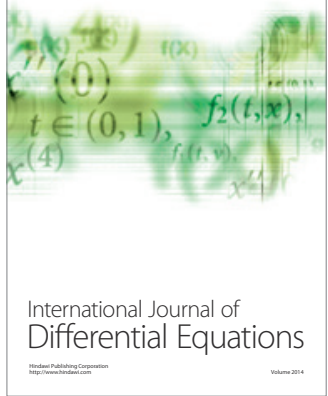
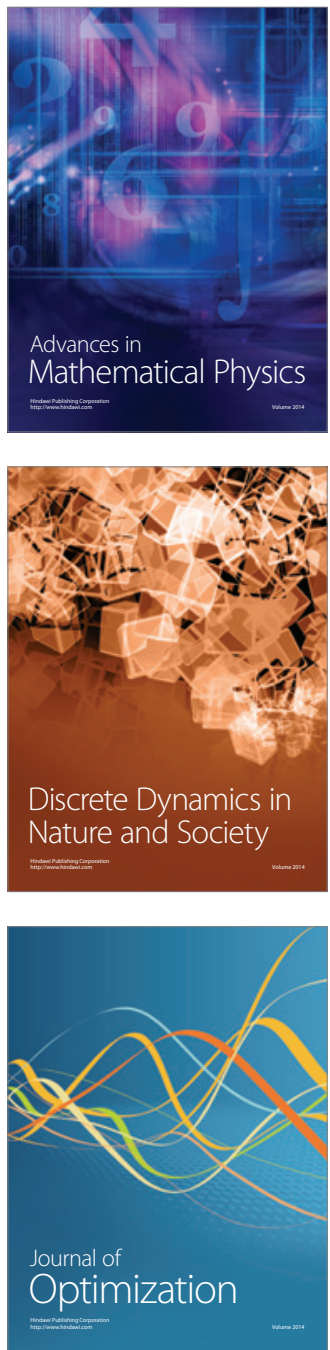